\title{
Electron-phonon coupling in surface electronic states on $\mathrm{Be}(10 \overline{10})$
}

\author{
I. Yu. Sklyadneva, ${ }^{1,2,3,4}$ R. Heid, ${ }^{3}$ P. M. Echenique,,${ }^{1,5,6}$ K.-P. Bohnen, ${ }^{3}$ and E. V. Chulkov ${ }^{1,5,6}$ \\ ${ }^{1}$ Donostia International Physics Center (DIPC), 20018 San Sebastián/Donostia, Basque Country, Spain \\ ${ }^{2}$ Institute of Strength Physics and Materials Science, pr. Academicheskii 2/1, 634021 Tomsk, Russia \\ ${ }^{3}$ Karlsruher Institut für Technologie, Institut für Festkörperphysik, D-76021 Karlsruhe, Germany \\ ${ }^{4}$ Tomsk State University, 634050, Tomsk, Russia \\ ${ }^{5}$ Departamento de Física de Materiales, Facultad de Ciencias Químicas, UPV/EHU, Apdo. 1072, 20080 San Sebastián/Donostia, \\ Basque Country, Spain \\ ${ }^{6}$ Centro de Física de Materiales CFM - Materials Physics Center MPC, Centro Mixto CSIC-UPV/EHU, 20018 San Sebastian/Donostia, Spain
}

(Received 25 October 2010; revised manuscript received 3 April 2011; published 26 May 2011)

\begin{abstract}
We present an $a b$ initio study of the electron-phonon interaction in surface electronic states on $\operatorname{Be}(10 \overline{1} 0)$. The calculations based on density-functional theory were carried out using a linear-response approach and a mixed-basis pseudopotential method. It is shown that the strength of the electron-phonon coupling is sensitive to the energy and momentum position of an electronic state and varies from 0.16 to 0.54 . The difference in the electron-phonon interaction of two Shockley-type surface states at $\overline{\mathrm{A}}$ can be understood on the basis of their different energy positions in the projected band gap. Previous experiments addressing the electron-phonon coupling strength of these two surface states are discussed in light of our theoretical findings.
\end{abstract}

DOI: 10.1103/PhysRevB.83.195437

PACS number(s): 63.20.kd, 68.47.De, 73.20.-r

\section{INTRODUCTION}

The electron-phonon (e-ph) interaction plays an important role in understanding various properties of metals from electrical conductivity to superconductivity. ${ }^{1,2}$ In particular, the $e$-ph coupling causes the enhancement of the effective electron mass and the renormalization of the electronic specific heat. It also contributes to transport properties and lifetimes of excited electrons, which are essential in many processes such as photochemical and catalytical reactions ${ }^{3-10}$ The strength of the interaction is given in terms of a dimensionless $e$-ph coupling parameter $\lambda$. In surface electronic states, $\lambda$ can be measured using the angle-resolved photoemission (ARPES) giving detailed information on the energy and momentum dependence of the interaction. ${ }^{10-15}$ Recent advances in highresolution angle-resolved spectroscopy have stimulated the study of the $e$-ph interaction at various metal surfaces. ${ }^{14-23}$ The first method of extracting the $e$-ph coupling parameter uses the temperature dependence of the linewidths of quasiparticle peaks measured with ARPES. At temperatures much higher than the Debye temperature $\left(T \gg T_{D}\right)$, this relation becomes linear and independent of phonon spectrum details. Thus, if $T_{D}$ is not high, $\lambda$ can be deduced from the slope of the width versus temperature data. ${ }^{1}$ Otherwise, the $e$-ph parameter can be obtained by fitting the experimental data with a simple model for the phonon density of states. ${ }^{10}$ This method, with the assumption that the predominant contribution to the linewidth temperature dependence can be attributed to the $e$-ph coupling, has been widely used ${ }^{11,16-21}$ to obtain $\lambda$ for many surface electronic states except for those located close to the Fermi energy $\left(\epsilon_{F}\right)$. Another approach is based on the direct extraction of the real part of the self-energy from ARPES measurements of a renormalized dispersion near the Fermi level. The strength of $e$-ph coupling can be obtained either from the slope of the real part at $\epsilon_{F}$ and at $T \ll T_{D}$ or by subsequent fitting of the experimentally derived data with a simple model. ${ }^{22-24}$ For metals with a smooth electronic density of states around the Fermi level, there is also a possibility of a direct extraction of the $e$-ph spectral (Eliashberg) function from the self-energy.
In this case, low-temperature measurements are not required and assumptions on phonon modes do not have to be made. ${ }^{24}$

Beryllium surfaces have attracted interest because of its unusual physical properties with respect to bulk Be. ${ }^{19-30}$ For instance, while bulk Be with a low electronic density of states (DOS) at the Fermi energy is almost a semimetal, its surfaces are metallic due to the electronic states that exist in energy gaps of the bulk projected band structure and give rise to a high density of states at $\epsilon_{F}{ }^{26,29}$ The latter was used to justify the larger $\lambda$ value at the surface Fermi level ${ }^{19,22-24}$ compared to that $(\lambda=0.21-0.24)$ obtained at $\epsilon_{F}$ of bulk Be. ${ }^{1,31}$ As regards the $\mathrm{Be}(10 \overline{10})$ surface, a great deal of experimental information is now available on the $e$-ph coupling of two Shockley-type surface electronic states at the surface Brillouin zone (SBZ) boundary (the $\overline{\mathrm{A}}$ point). Several experimental studies ${ }^{19-21,24}$ using both the temperature dependence of the photoemission linewidth and the renormalization of the surface-state dispersion at $\epsilon_{F}$ revealed that the strength of the $e$-ph interaction in these surface states was different.

Here we present a detailed theoretical analysis of the electron-phonon interaction in surface electronic states on $\mathrm{Be}(10 \overline{10})$. Though all the surface states are considered, much attention is devoted to the Shockley-type surface electronic states at $\overline{\mathrm{A}}$. The strength of the $e$-ph coupling is presented as a function of electron energy and momentum. We have also evaluated the contributions to the $e$-ph interaction coming from different phonon modes. In particular, the important role of surface phonons is emphasized. Some of these vibrations are much softer (stiffer) than the bulk ones and couple in a very efficient way to electrons, giving a large contribution to the $e$-ph coupling. It is shown that $\lambda$, being sensitive to the energy and momentum position of an electronic state, ranges from 0.16 to 0.54 . The strength of the $e$-ph interaction obtained for the lower surface electronic state at $\bar{A}$ is in perfect agreement with the value extracted from ARPES measurements. ${ }^{21}$ At the same time, the experimental and theoretical data on the $e$-ph coupling in the upper surface electronic state at $\overline{\mathrm{A}}$ differ substantially. Therefore, we present 
a detailed discussion of the striking discrepancy between the calculated and experimentally derived values.

The paper is organized as follows. A short outline of the calculation method is given in Sec. II. Section III contains both the results for the electron-phonon coupling and the discussion of the calculated and experimentally derived data. Finally, in Sec. IV the conclusions are drawn.

\section{DETAILS OF THE CALCULATION}

The first-principles calculations were performed within the density-functional theory and the local-density approximation for the exchange-correlation potential. ${ }^{32,33}$ The dynamical properties were obtained using the density-functional perturbation theory. ${ }^{34}$ The electron-ion interaction in $\mathrm{Be}$ atoms was described by a norm-conserving pseudopotential ${ }^{35}$ that included nonlinear core corrections. ${ }^{36}$ The electronic wave functions were expanded in a set of plane waves up to a kinetic energy cutoff of $22 \mathrm{Ry}$. To integrate over the surface Brillouin zone, the Gaussian smearing technique was used with a width of $0.2 \mathrm{eV}$ and a $(24 \times 16)$ special point mesh in the SBZ. The $\operatorname{Be}(10 \overline{1} 0)$ surface was simulated by repeated 24-layer $\mathrm{Be}(10 \overline{1} 0)$ slabs separated by a vacuum region of six atomic layers sufficient to decouple the two surfaces of a slab. Atomic positions inside the $\mathrm{Be}(10 \overline{1} 0)$ layers maintain the symmetry of the bulk and are fixed at the theoretical bulk lattice parameters, which minimize the total energy, $a=4.186$ a.u. and $c / a=1.5822$. The deviation from the experimental values ${ }^{37}$ taken at room temperature, $a=4.32$ a.u. and $c / a=1.568$, is about $3 \%$.

A central quantity of the $e$-ph interaction is the Eliashberg spectral function ${ }^{38} \alpha^{2} F\left(\epsilon_{i}, \mathbf{k} ; \omega\right)$, which measures the contribution of phonons with frequency $\omega$ to the $e$-ph coupling between an initial electronic state with momentum $\mathbf{k}$ and energy $\epsilon_{i}$ and all possible final states that differ in energy by $\hbar \omega .{ }^{1}$ The electronic state-dependent Eliashberg spectral function corresponding to phonon emission $(E)$ and absorption $(A)$ processes is defined as

$$
\begin{aligned}
& \alpha^{2} F^{E(A)}\left(\epsilon_{i}, \mathbf{k} ; \omega\right) \\
& =\sum_{\mathbf{q}, v, f} \delta\left(\epsilon_{i}-\epsilon_{f} \mp \omega_{\mathbf{q} \nu}\right) \\
& \quad \times\left|g^{i, f}(\mathbf{k}, \mathbf{q}, v)\right|^{2} \delta\left(\omega-\omega_{\mathbf{q} v}\right),
\end{aligned}
$$

where $g^{i, f}(\mathbf{k}, \mathbf{q}, v)$ is the $e$-ph matrix element between the initial $(i)$ and final $(f)$ electronic states. The "-" and "+" signs in the $\delta$ function correspond to phonon emission $E$ and absorption $A$, respectively. The sum is carried out over final electronic states and all phonon modes $(\mathbf{q} v)$.

The $e$-ph matrix element is expressed in terms of microscopic quantities as

$$
g^{i, f}(\mathbf{k}, \mathbf{q}, v)=\sqrt{\frac{\hbar}{2 \omega_{\mathbf{q} v}}} \sum_{\kappa a} \frac{\epsilon_{\kappa a}^{\mathbf{q} v}}{\sqrt{M_{\kappa}}}\left\langle f\left|\delta_{\kappa a}^{\mathbf{q}} V\right| i\right\rangle .
$$

$M_{\kappa}$ is the mass of the $\kappa$ th atom in the unit cell, and $\epsilon_{\kappa a}^{\mathbf{q} v}$ is the normalized eigenvector of the phonon mode $(\mathbf{q} v)$. The finalstate momentum is determined by $\mathbf{k}_{f}=\mathbf{k}+\mathbf{q}+\mathbf{G}$, where $\mathbf{k}$ is the momentum of the initial state and $\mathbf{G}$ is a reciprocal-lattice vector. Finally, $\delta_{\kappa a}^{\mathbf{q}} V$ denotes the first-order change of the total crystal potential with respect to the displacement of atom $\kappa$ in the $a$ direction, which is available as a result of a phonon calculation.

Since the $e$-ph matrix elements for a fixed electronic state display rather significant variations throughout the SBZ, the summation in Eq. (1) over wave vectors requires a dense mesh of $\mathbf{q}$ points. So, we first calculated the electron-phonon matrix elements using a $(12 \times 8) \mathbf{q}$ point mesh. Then, to check the convergence, the summation was carried out on a denser $(48 \times$ 32) q point mesh. For the additional $\mathbf{q}$ vectors, the matrix elements were calculated using a Fourier interpolation scheme for the change of the self-consistent potential with respect to atomic displacements as well as for the dynamical matrices. The $\delta$ function with electron energies was approximated by the first-order Hermite-Gaussian function with a smearing width in the range of $0.01-0.03 \mathrm{Ry}$.

The electronic state-dependent parameter measuring the coupling strength is just the first reciprocal moment of the Eliashberg function:

$$
\lambda\left(\epsilon_{i}, \mathbf{k}\right)=\int_{0}^{\omega_{m}} \frac{\alpha^{2} F^{E}\left(\epsilon_{i}, \mathbf{k} ; \omega\right)+\alpha^{2} F^{A}\left(\epsilon_{i}, \mathbf{k} ; \omega\right)}{\omega} d \omega .
$$

Here $\omega_{m}$ is the maximum phonon frequency. If $\lambda\left(\epsilon_{i}, \mathbf{k}\right)$ is averaged over all initial electronic states on the Fermi surface, one obtains the usual isotropic $e$-ph coupling constant ${ }^{39}$ $\lambda\left(\epsilon_{F}, \mathbf{k}_{F}\right)$.

\section{RESULTS AND DISCUSSION}

\section{A. Electronic and dynamical properties}

In principle, the (1010) surface of hcp metals can be terminated with either a short first interlayer spacing or a long one, which differ by a factor of 2 . In the case of $\operatorname{Be}(10 \overline{1} 0)$, only the short termination was shown both experimentally and theoretically to be stable. ${ }^{29,40}$ Therefore, this geometry was assumed in the present study. At first, we calculated the relaxed interlayer spacings by minimizing forces between atomic layers. An oscillatory relaxation was found with a $21.5 \%$ contraction of the topmost interlayer distance and the expansion of the second one by $5.3 \%$ with respect to the bulk. These values as to the sign and magnitude of the relaxation are consistent with the experimental data of $-25(-4 /+$ $3) \%$ and $5(-4 /+3) \%$ obtained by low-energy electrondiffraction (LEED) intensity analysis. ${ }^{40}$ The calculated values are also in agreement with other $a b$ initio pseudopotential results, $\Delta d_{12}=-24.5 \%$ (Ref. 41 ) and $\Delta d_{12}=-21.6 \%$ (Ref. 42).

The surface electronic states obtained for a 24-layer $\operatorname{Be}(10 \overline{1} 0)$ slab are shown by black lines in Fig. 1, while the shaded background represents the bulk electronic bands projected onto the (1010) plane. The surface-state positions in the figure are the average between the energies of bonding and antibonding states formed by the interaction of the surface states on both sides of the slab. The energy difference is usually negligible $(<0.06 \mathrm{eV})$. Only in the vicinity of the $\bar{\Gamma}$ point, where the surface resonance has a large penetration depth, does the difference increase up to $0.18 \mathrm{eV}$. We have summarized the surface electronic state energies both predicted theoretically and observed experimentally in Table I for comparison. The 


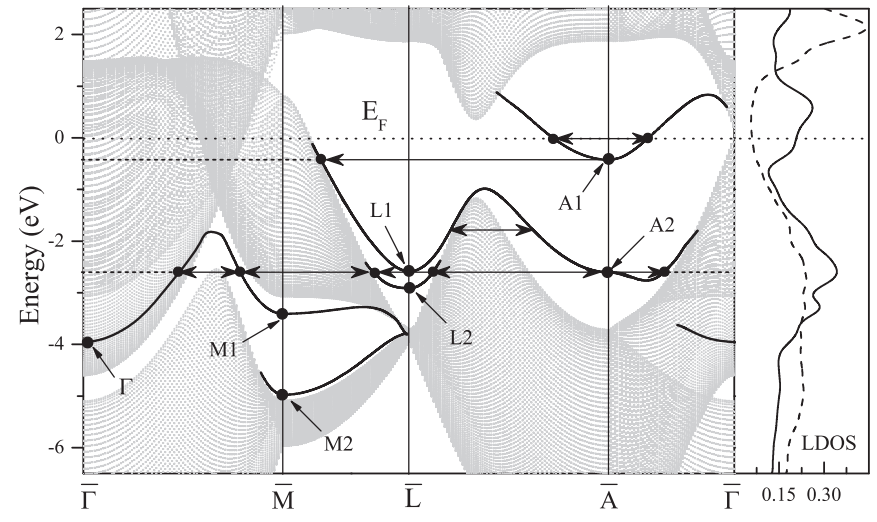

FIG. 1. Calculated surface electronic states (black lines) for a 24-layer Be(1010) slab. The gray background gives bulk Be bands projected onto the two-dimensional Brillouin zone. Phonon-mediated transitions for the surface electronic states at $\overline{\mathrm{A}}$ are shown by arrows and dashed lines. The right panel shows the local electronic density of states for the surface (solid line) and central (dashed line) layers of the slab.

right panel of Fig. 1 shows the local density of states (LDOS) for the surface (solid line) and central (dashed line) layers of the slab. As expected, they differ markedly. In the surface layer LDOS, a peak appears between -0.5 and $1 \mathrm{eV}$ due to the A1 surface electronic state. As a result, at the Fermi energy the LDOS in the surface layer is three times larger than that in the bulklike central layer. ${ }^{29,43}$

Although the calculated phonon-dispersion curves of the $\operatorname{Be}(10 \overline{1} 0)$ surface have already been reported for the $\overline{\Gamma A}$ and $\overline{\Gamma M}$ symmetry directions, ${ }^{41,44}$ we show the phonon spectrum in Fig. 2 to facilitate the discussion of vibrational modes contributing to the $e$-ph coupling. The phonon-dispersion curves in Fig. 2 correspond to a 100-layer $\mathrm{Be}(10 \overline{10})$ slab to decouple those surface vibrations that penetrate deeply into the bulk. The dynamical matrices of the slab were built combining calculated surface force constants to ab initio bulk force constants obtained from a similar bulk calculation using a $(12 \times 12 \times 8) \mathbf{q}$ point mesh. The phonon spectrum is shown along all high-symmetry directions because the surface phonon modes at the SBZ boundary ( $\overline{\mathrm{MLA}})$ turned out to be the most important in the $e$-ph coupling. Since the slabs used in the calculation were asymmetric with one relaxed surface and the other surface corresponding to the ideal bulk-truncated case, the phonon spectrum contains surface modes related to both types of surfaces. The surface mode frequencies

TABLE I. Summary of surface electronic state energies at the high-symmetry points (in $\mathrm{eV}$ relative to $\epsilon_{F}$ ).

\begin{tabular}{lccc}
\hline \hline & Expt. $^{29}$ & Expt. $^{20}$ & This work \\
\hline M1 & -3.6 & $-3.47 \pm 0.05$ & -3.41 \\
M2 & & $-4.90 \pm 0.06$ & -4.98 \\
L1 & $-2.45 \pm 0.03$ & -2.59 \\
L2 & & $-2.80 \pm 0.02$ & -2.91 \\
A1 & -0.33 & $-0.416 \pm 0.02$ & -0.43 \\
A2 & -2.7 & $-2.73 \pm 0.04$ & -2.6 \\
$\Gamma$ & -4.05 & $-4.07 \pm 0.04$ & -3.96 \\
\hline \hline
\end{tabular}

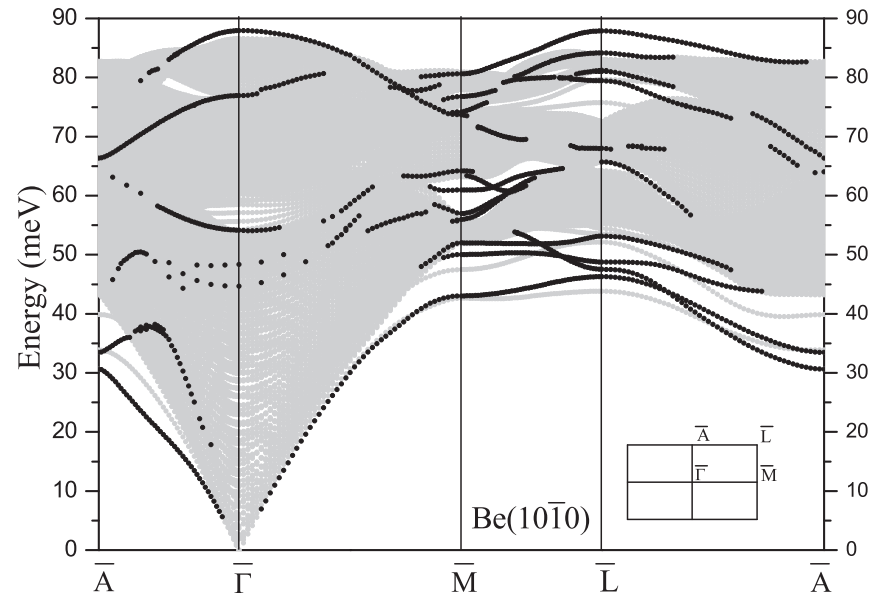

FIG. 2. Calculated phonon-dispersion curves for a 100-layer $\mathrm{Be}(10 \overline{10})$ slab. The phonon spectrum contains surface modes related to both relaxed (black dots) and ideal bulk-truncated (gray dots) surfaces.

at high symmetry points are given in Table II. We have summarized both the calculated values and those observed experimentally. ${ }^{30}$

The first $a b$ initio calculation of the $\mathrm{Be}(10 \overline{10})$ phonon spectrum was done using a plane-wave-based densityfunctional perturbation theory. ${ }^{41}$ The authors were able to assign most surface modes observed experimentally ${ }^{30}$ except for the feature at $63.3 \mathrm{meV}$ at $\bar{\Gamma}$. It was identified to be caused by an enhanced vibrational density of states and not by a surface localized mode. Indeed, there are many surface resonances with small wave vectors between 60 and $70 \mathrm{meV}$. Some of them are characterized by inplane vibrations of atoms along close-packed rows (the $x$ direction). As is well known, a hcp (1010) surface can be considered as a stepped one with the first layer consisting of parallel closed-packed rows of atoms like an fcc (110) surface. But most of the resonances are polarized along the normal to the surface (vertically polarized). In general, the agreement between both ab initio calculations is quite good. However, unlike the previous study, we show in Fig. 2 more surface resonances. As one can see, the $\mathrm{Be}(10 \overline{10})$ surface is characterized by the abundance of surface localized lattice vibrations. In spite of this fact, one can outline three important groups of modes for the analysis of the $e$-ph coupling. The first

TABLE II. Surface mode frequencies (in meV) at high-symmetry points.

\begin{tabular}{lcccccccccc}
\hline \hline & $\bar{\Gamma}$ & & & \multicolumn{5}{c}{$\overline{\mathrm{A}}$} \\
\hline Expt. $^{30}$ & 43.0 & & 55.2 & & & & & 33.0 & \\
Theory $^{41}$ & 45.9 & & 52.9 & 73.9 & 84.8 & & 26.4 & 32.3 & 63.7 \\
This work & 44.7 & 48.3 & 54.2 & 77.2 & 88.2 & & 30.6 & 33.6 & 66.5 \\
& $M$ & & & & & & & & \\
Expt. $^{30}$ & 40.0 & 47.0 & & & & & & & \\
Theory $^{41}$ & 41.4 & 49.8 & & 54.3 & 56.0 & 61.1 & 68.7 & 69.4 & 76.5 \\
This work & 43.2 & 50.3 & 52.3 & 57.2 & 61.5 & 64.3 & 74.0 & 76.9 & 80.9 \\
& $\bar{L}$ & & & & & & & & \\
This work & 46.5 & 47.7 & 48.9 & 53.4 & 68.3 & 79.7 & 81.5 & 84.4 & 88.2 \\
\hline \hline
\end{tabular}


TABLE III. Calculated electron-phonon coupling parameter $\lambda(\epsilon, \mathbf{k})$ for surface electronic states at the symmetry points. The uncertainty arising from the employed interpolation scheme is 0.02 or less.

\begin{tabular}{llll}
\hline \hline & $\lambda$ & & $\lambda$ \\
\hline A1 & 0.16 & L1 & 0.34 \\
A2 & 0.44 & L2 & 0.41 \\
M1 & 0.39 & $\Gamma$ & 0.23 \\
M2 & 0.25 & & \\
\hline \hline
\end{tabular}

one is formed by two lower surface modes propagating along the $\overline{\Gamma A}$ direction. They are vertically polarized and localized in the second (the lowest one) and in the first layer. Another surface mode, an optical one with an energy of $66.5 \mathrm{meV}$ at $\overline{\mathrm{A}}$, is characterized by the in-plane motion of the firstand the third-layer atoms along the close-packed rows. The second group is formed by mainly vertically polarized surface vibrations that split off and lie below the bulk phonons or at the edge of bulk vibrations along the SBZ boundary. Their energies vary in the range from 30 to $55 \mathrm{meV}$. Among these modes, the lowest one in the $\overline{\mathrm{ML}}$ direction, which becomes the second mode on approaching the $\overline{\mathrm{A}}$ point, is the so-called Rayleigh mode with the polarization in the sagittal plane. The third group, at energies $\geqslant 75 \mathrm{meV}$, consists mostly of the in-plane lattice vibrations of both surface and deeper-lying atomic layers up to the fourth one. The most prominent modes of this group are those located above the bulklike lattice vibrations. The first one, around the $\bar{\Gamma}$ point, is associated with the motion in the upper four layers perpendicular to the close-packed atomic rows (the $y$ direction). The second phonon branch, at the SBZ boundary, is characterized by vibrations of atoms in two perpendicular directions: the motion in the topmost layer occurs along the close-packed rows whereas the subsurface atoms move perpendicular to them.

\section{B. Electron-phonon coupling}

The hcp (1010) surface is characterized by two wide bulk projected band gaps. Two surface electronic states are located in the lower one and four states lie inside the upper gap (see Fig. 1). We have calculated the $e$-ph coupling parameter as a function of electron energy and momentum for all of them. The values of $\lambda(\epsilon, \mathbf{k})$ for the surface electronic states at the symmetry points are shown in Table III.

\section{Surface electronic states at $\overline{\mathrm{M}}, \overline{\mathrm{L}}$, and $\bar{\Gamma}$}

We first address the $e$-ph coupling in the surface states at $\overline{\mathrm{L}}, \overline{\mathrm{M}}$, and $\bar{\Gamma}$. Their $e$-ph spectral functions are similar to each other and mainly differ in the size of a high-energy peak above $80 \mathrm{meV}$, which is very pronounced for the states M1 and L2 while small for the other ones (M2, L1, and $\bar{\Gamma})$. Figure 3 shows $\alpha^{2} F(\omega)$ for M1 (solid line) and M2 (dashed line) as two representatives. Since the contributions from phonon emission and absorption processes almost coincide, only the averaged Eliashberg spectral functions are shown. The spectra are similar except for the high-energy part, which is largely determined by contributions from surface localized vibrations characterized by in-plane motion of atoms (the third

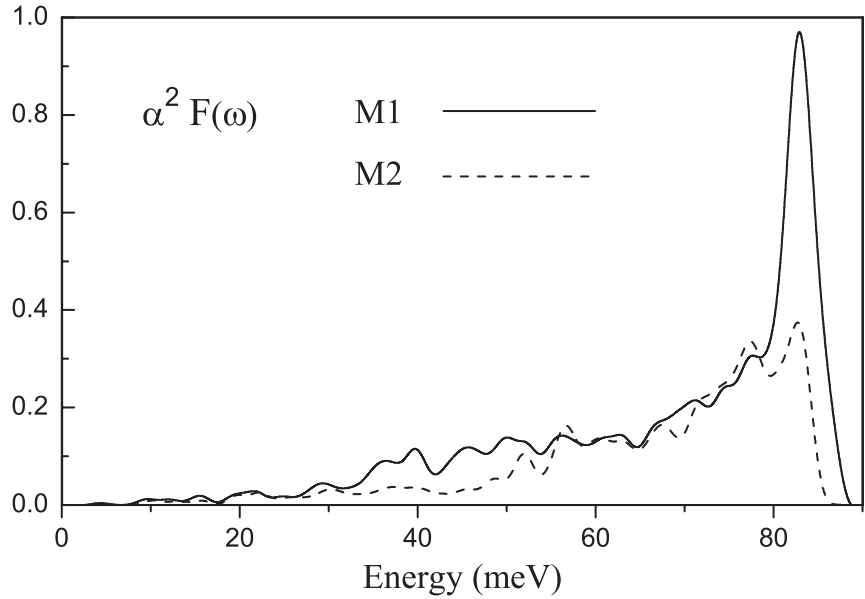

FIG. 3. Electron-phonon Eliashberg spectral function $\alpha^{2} F(\omega)$ for M1 (solid line) and M2 (dashed line) surface electronic states.

group of surface modes discussed in Sec. III A). These modes couple most effectively to the well-localized states M1 and L2, enhancing their $e$-ph coupling strengths. Besides, M1 is characterized by an exceptional role of intraband transitions that amount to $\sim 45 \%$ of the total $e$-ph coupling.

The $e$-ph interaction for the second group of states, M2, L1, and $\bar{\Gamma}$, is dominated by phonon-mediated transitions to bulk electronic states. The intraband scattering provides $\sim 15 \%$ of the total contribution to $\lambda(\epsilon, \mathbf{k})$. Because of the weak highfrequency peak in $\alpha^{2} F(\omega)$, they exhibit smaller $\lambda(\epsilon, \mathbf{k})$ than M1 and L2. However, for L1 a substantial contribution coming from the coupling to other surface electronic states (see Fig. 1) raises $\lambda_{\mathrm{L} 1}$ closer to the strength of the $e$-ph interaction at L2 or M1.

\section{Surface electronic states at $\overline{\mathbf{A}}$}

We now proceed to the Shockley-type surface electronic states around $\overline{\mathrm{A}}$ which spectral functions at $\overline{\mathrm{A}}$ are shown in Figs. 4(a) and 4(b). The figure also gives the local phonon densities of states for the surface (solid line) and central (dashed line) layers of a 24-layer slab, Fig. 4(c). The spectral functions can be analyzed in terms of low-energy (up to $\sim 62 \mathrm{meV}$ ) and high-energy parts. The latter forms either a broad feature (A2) or two peaks (A1), and is mainly associated with contributions from in-plane lattice vibrations both bulklike and localized at the surface. The lower-energy part shows a multipeak structure resulting from the interaction of electrons with surface phonon modes, including Rayleigh modes, which split off the bulklike continuum at the SBZ boundary (see Fig. 2).

a. The lower surface state at $\overline{\mathrm{A}}$. At first, we concentrate on the lower surface state, A2, which disperses toward $\epsilon_{F}$ along $\overline{\mathrm{AL}}$ while in the $\overline{\mathrm{A} \Gamma}$ direction it enters gradually the bulk electronic bands and loses its surface character. A2 is mainly localized in the subsurface layer, where its orbital composition is determined by $s$ and $p_{z}$ contributions whereas at the surface it is purely $p_{y}$ in character. The $e$-ph coupling parameter calculated at $\overline{\mathrm{A}}, \lambda_{\mathrm{A} 2}=0.44 \pm 0.02$, is in good agreement with the value of $\lambda_{\mathrm{A} 2}=0.49 \pm 0.04$ extracted from temperature-dependent measurements of the photoemission 

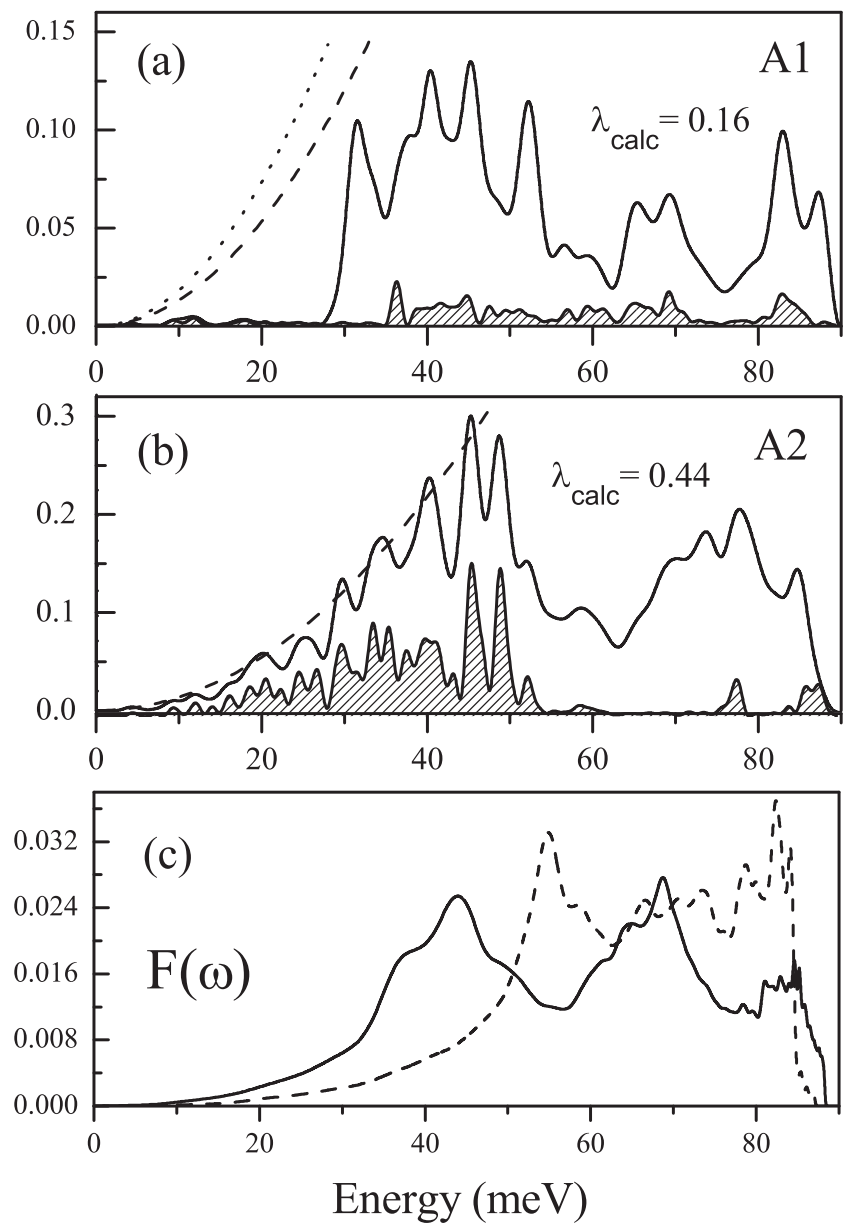

FIG. 4. (a,b) Electron-phonon Eliashberg spectral function $\alpha^{2} F(\omega)$ (solid line) for A1 (a) and A2 (b) surface electronic states on $\mathrm{Be}(10 \overline{1} 0)$. The contribution corresponding to the intraband scattering is shown by a shaded area. Dashed and dotted lines show the behavior of the spectral function at small energies when the Debye model for phonons is used, $\alpha^{2} F(\omega)=\lambda\left(\omega / \omega_{m}\right)^{2}$, with $\hbar \omega_{m}=60 \mathrm{meV}$ from LEED measurements ${ }^{40}$ and $\lambda$ extracted from the fitting of the experimental linewidth vs temperature data. The dashed lines correspond to $\lambda \approx 0.48$ (a) and $\lambda \approx 0.49$ (b) obtained by fitting ${ }^{21}$ over a full temperature range of $45-700 \mathrm{~K}$. The dotted line (a) corresponds to $\lambda \approx 0.66$ from fitting ${ }^{20,21}$ over a limited temperature range of 300-700 K. (c) Local densities of phonon states, $F(\omega)$, for the surface (solid line) and central (dashed line) layers of a 24-layer slab.

linewidth $^{21}$ fitted with a Debye model. The energy and momentum dependence of $\lambda(\epsilon, \mathbf{k})$ along $\overline{\mathrm{A} \Gamma}$ and $\overline{\mathrm{AL}}$ symmetry directions is shown in Fig. 5 (the lower panel).

An analysis of initial and final electronic states in the $e$-ph scattering processes showed that a large part of the phonon-mediated transitions contributing to $\lambda_{\mathrm{A} 2}$ comes from the intraband scattering, which amounts to $30 \%$ [the shaded area in Fig. 4(b)]. That is expected because of a small dispersion of the state in the vicinity of the $\overline{\mathrm{A}}$ point, especially taking into account a large maximum phonon energy for Be. A substantial contribution $(\sim 20 \%)$ also comes from the coupling to the surface electronic states around the $\overline{\mathrm{L}}$ point, A2 $\Leftrightarrow \mathrm{L} 1(\mathrm{~L} 2)$, and in the $\overline{\Gamma M}$ symmetry direction. Along $\overline{\Gamma L}$
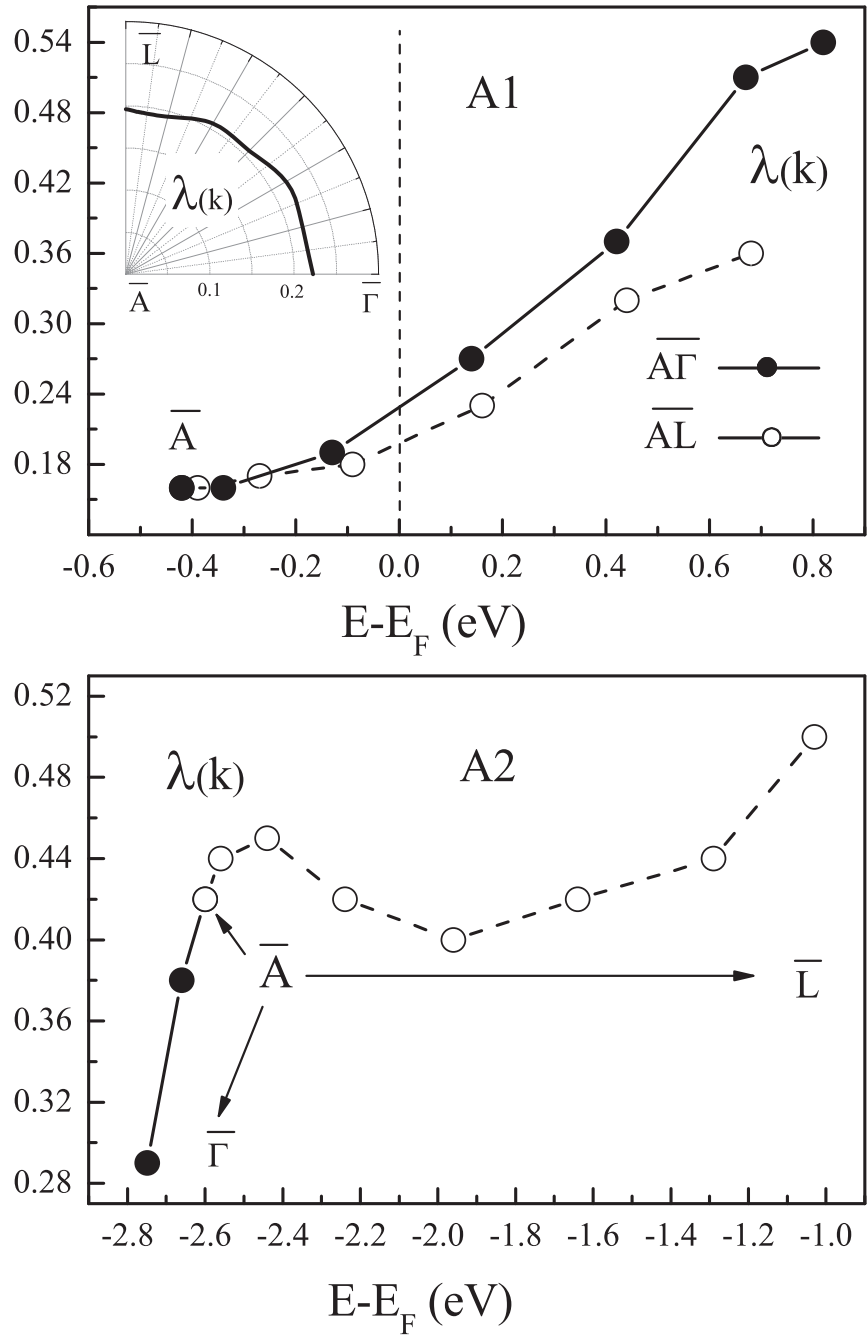

FIG. 5. Electron-phonon coupling parameter $\lambda(\mathbf{k})$ as a function of binding energy for electronic states in the A1 and A2 surface bands along two symmetry directions, $\overline{\mathrm{A} \Gamma}$ (solid lines with full circles) and $\overline{\mathrm{AL}}$ (dashed lines with open circles). The inset in the upper panel shows $\lambda_{\mathrm{A} 1}\left(\epsilon_{F}\right)$ as a function of electron momentum along a surface Fermi line from the $\overline{\mathrm{A} \Gamma}$ to $\overline{\mathrm{AL}}$ direction.

the binding energy of the A2 state decreases, but the strength of the $e$-ph coupling does not change significantly, ranging from 0.4 to 0.5 . Simultaneously, the state assumes gradually a $p_{x}$ character in the subsurface layer where it is mainly localized while the state symmetry in the topmost layer, $p_{y}$, is kept. The character of transitions that contribute to the $e$-ph coupling also changes. With decreasing binding energy, the intraband scattering diminishes while the interband junctions to the L1 surface electronic states begin to dominate. The latter can also be regarded as intraband ones because the L1 surface band can be considered as a continuation of A2.

b. The upper surface state at $\overline{\mathrm{A}}$. The upper surface state, A1, is connected to a surface band that disperses upward in all directions and builds a small Fermi surface with almost circular shape. Its energy and momentum dependence of $\lambda(\epsilon, \mathbf{k})$ are shown in Fig. 5 (the upper panel). The inset also gives the variation of $\lambda_{\mathrm{A} 1}\left(\epsilon_{F}\right)$ when going along the Fermi contour from the $\overline{\mathrm{A} \Gamma}$ to the $\overline{\mathrm{AL}}$ direction. It shows that the 
calculated strength of the $e$-ph coupling is quite small both at $\overline{\mathrm{A}}$ $\left(\lambda_{A 1}=0.16\right)$ and at the Fermi level, where it exhibits a small directional anisotropy $\left[\lambda_{\mathrm{A} 1}\left(\epsilon_{F}, \mathbf{k}\right)=0.18-22\right]$. However, on moving further away from the $\overline{\mathrm{A}}$ point into the region of unoccupied states, the $e$-ph coupling strength increases and develops a sizable directional anisotropy. On moving to the SBZ center, the energy dependence is stronger and $\lambda(\epsilon, \mathbf{k})$ increases up to 0.5 , while in the $\overline{\mathrm{AL}}$ direction, $\lambda(\epsilon, \mathbf{k})=0.36$ at the same binding energy of $\sim 0.67 \mathrm{eV}$. The enhancement is determined, for the most part, by the intraband scattering. The number of intraband transitions increases and, at $\epsilon_{F}$, their contribution to $\lambda(\epsilon, \mathbf{k})$ amounts to $\sim 30 \%$ as compared to $10 \%$ at $\overline{\mathrm{A}}$. In addition, for the surface states in the $\overline{\mathrm{A} \Gamma}$ symmetry direction, some of the intraband transitions are provided by low-energy surface phonon modes from the $\overline{\Gamma A}$ symmetry direction. The corresponding sharp peak appears in the spectral function at 20-25 meV (see Fig. 6). The contribution of these phonons to the strength of the $e$-ph coupling is quite large due to the weighting of $\alpha^{2} F(\omega)$ by $1 / \omega$ in the expression for $\lambda$
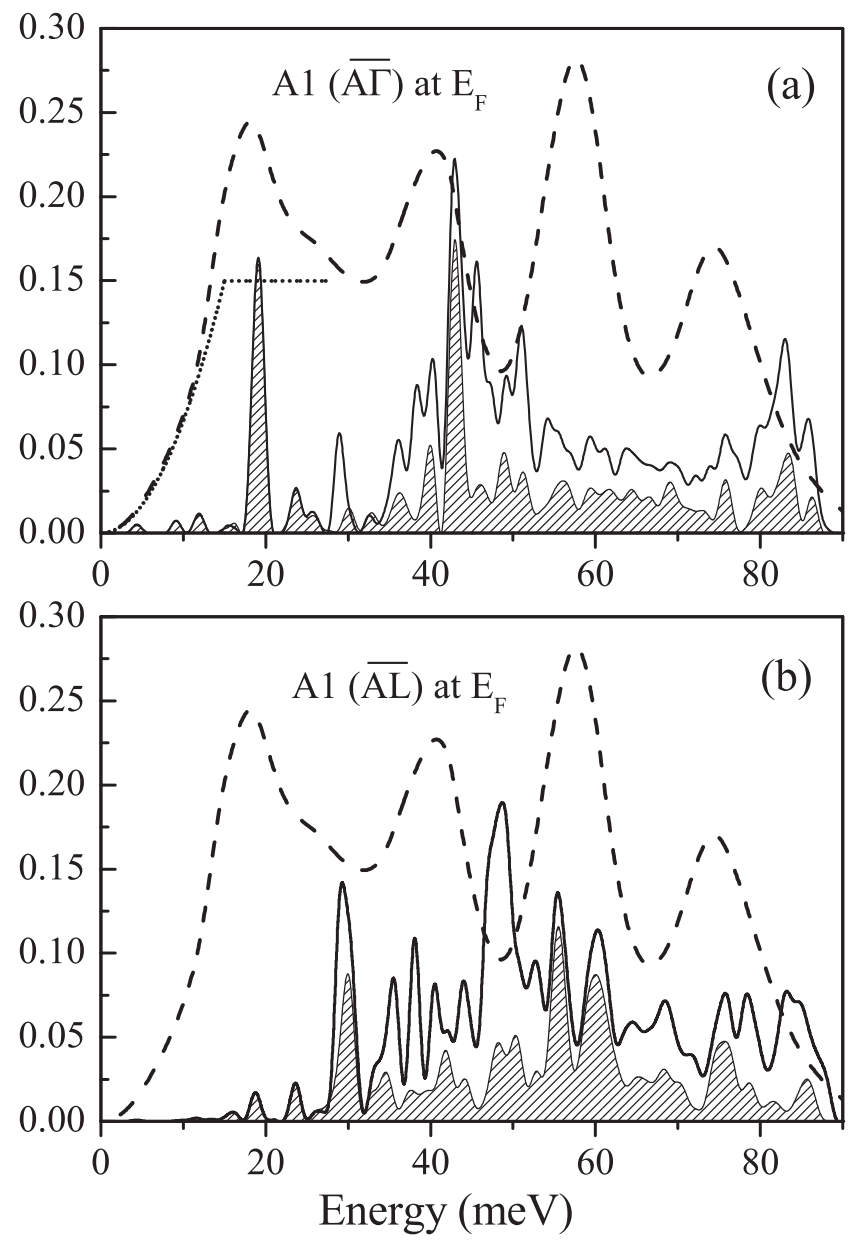

FIG. 6. Electron-phonon Eliashberg spectral function for the A1 surface electronic state at $\epsilon_{F}$ in the $\overline{\mathrm{A} \Gamma}$ (a) and $\overline{\mathrm{AL}}$ (b) symmetry directions: the calculated $\alpha^{2} F(\omega)$ (solid line) with the contribution from the intraband scattering shown by a shaded area and the function extracted from photoemission measurements ${ }^{24}$ (dashed line). The small energy behavior of the constraint function used to obtain $\alpha^{2} F(\omega)$ from experimental data is also shown by a dotted line in panel (a).
[Eq. (3)]. For the surface states in the $\overline{\mathrm{AL}}$ symmetry direction as well as at $\overline{\mathrm{A}}$ [Fig. 4(a)], the spectral function is negligible up to nearly $30 \mathrm{meV}$.

c. Comparison of $A 1$ and A2. Our calculations outlined above reveal that the two surface states at $\overline{\mathrm{A}}$ exhibit rather different coupling strengths, with $\lambda_{\mathrm{A} 1}=0.16$ and $\lambda_{\mathrm{A} 2}=0.44$. Here we indicate the main factors that result in such different coupling strengths.

The main reason for the small value of $\lambda_{\mathrm{A} 1}$ is the position of the A1 state in the middle of a wide projected band gap (see Fig. 1). As a consequence, only phonons with large momenta can couple. These are predominantly those located close to the $\overline{\mathrm{AL}}$ and, partially, $\overline{\mathrm{ML}}$ symmetry direction. Coupling of A1 to modes along the $\overline{\Gamma A}$ direction is negligible because there are no final electronic states in the $\overline{\Gamma A}$ direction except for a few near the SBZ center. A further consequence is the absence of coupling to acoustic modes, which leads to a strong suppression of the $e$-ph spectral function below $\sim 30 \mathrm{meV}$. Furthermore, the coupling of A1 occurs almost exclusively to bulk states, because surface localized states at the binding energy of $\mathrm{A} 1$ are practically absent. All these properties conspire to result in a small $\lambda_{\mathrm{A} 1}$.

In contrast, A2 lies close to the lower band-gap edge, and their are many surface localized states available for coupling. Therefore, phonon modes with practically any wavelength contribute, and coupling to surface localized modes is much more probable.

We would like to note that despite the position of A2 close to the edge of the band gap, A2 is a well-localized state for which charge density does not penetrate deeply into the slab. The maximum of the A2 charge density is in the subsurface layer, while A1 is mainly localized in the topmost atomic layer. Thus, these surface states belong to different atomic chains of the $(10 \overline{10})$ surface, and the maximum charge density of the lower state lies at the minimum of the upper state.

The weak coupling of $\mathrm{A} 1$ is a direct consequence of the electronic surface band structure and of the surface phonon spectrum. As the present $a b$ initio approach describes both of these properties accurately, it is difficult to expect a large value of $\lambda(\epsilon, \mathbf{k})$ for $\mathrm{A} 1$.

\section{Discussion of measurements}

Three sets of studies have been devoted to the $e$-ph coupling of two Shockley-type surface electronic states at $\overline{\mathrm{A}}$ : (E1) Measurements of the temperature dependence of the photoemission linewidth at high temperatures. ${ }^{19,20}$ By fitting to the three-dimensional (3D) Debye model, a coupling constant of $\lambda_{\mathrm{A} 1}=0.665 \pm 0.03$ was found. Similar values were extracted for states of the A1 band at a binding energy of $\sim 0.2 \mathrm{eV}[\lambda=0.672 \pm 0.027$ in the $\overline{\mathrm{AL}}$ direction and $\lambda=0.642 \pm 0.031$ in the $\overline{\Gamma A}$ direction]. (E2) Measurements of the temperature dependence of the photoemission linewidth over an extended temperature range including low-temperature data. ${ }^{21}$ Both Shockley-type surface states at $\overline{\mathrm{A}}$ were examined. Fitting with an Einstein model gave $\lambda_{\mathrm{A} 1}=0.65 \pm 0.02$, while for A2 a 3D Debye phonon model was applied and resulted in $\lambda_{\mathrm{A} 2}=0.49 \pm 0.04$. (E3) High-resolution photoemission measurements were used to extract the real part of the electronic self-energy, from which the Eliashberg function was 
obtained using the maximum entropy method. ${ }^{24}$ The reported mass enhancement parameter for $\mathrm{A} 1$ at the Fermi energy (in the $\overline{\Gamma \mathrm{A}}$ direction) was $\lambda_{\mathrm{A} 1}\left(\epsilon_{F}\right)=0.68 \pm 0.08$.

While the study addressing A 2 obtained a coupling constant in very good agreement with the present calculation, all experiments on A1 agree on a very large value of $\lambda_{\mathrm{A} 1}$ of about 0.65-0.7, which is at striking variance with our theoretical analysis. As outlined in the previous subsection, the energetic position of the A1 state in the middle of a wide band gap naturally leads to a reduced coupling as compared to the A2 state. It is therefore rather difficult to understand how this state could acquire a coupling constant even $\approx 40 \%$ larger than $\mathrm{A} 2$.

Currently, the source of this discrepancy is unknown. However, all experiments rely on certain models and assumptions to extract $\lambda_{\mathrm{A} 1}$. In the following, we briefly examine the validity and potential shortcoming of these assumptions in light of our calculations.

Extraction of $\lambda_{\mathrm{A} 1}$ from temperature-dependent linewidth measurements relies on a model for the $e$-ph spectral function. Both models used in previous studies, a 3D Debye model in (E1) and an Einstein model in (E2), are inappropriate to describe the calculated $\alpha^{2} F(\omega)$ for A1 [Fig. 4(a)]: the spectral function, on the one hand, is strongly suppressed in the low-frequency part up to $\approx 30 \mathrm{meV}$, and, on the other hand, contains contributions from modes distributed over a wide frequency range of $\approx 30-90 \mathrm{meV}$. In contrast to the case of A1, the assumption of a Debye-like spectrum is appropriate in the case of the lower state A2 [see Fig. 4(b)].

The procedure to extract the Eliashberg function from highresolution photoemission measurements (E3) contains two steps. First, $\operatorname{Re} \Sigma_{\text {ph }}$ is extracted from the measured quasiparticle dispersion by subtracting a model bare dispersion. This step is known to depend very sensitively on the choice of the bare dispersion. Second, $\alpha^{2} F(\omega)$ is obtained from $\operatorname{Re} \Sigma_{\mathrm{ph}}$ by solving an integral equation via the maximum entropy method. This last step involved several physical constraints needed for proper convergence. In particular, the low-energy part of $\alpha^{2} F(\omega)$, which is crucial in the definition of $\lambda$, was taken to be Debye-like. The extracted $\alpha^{2} F(\omega)$ is compared in Fig. 6 with the calculated spectral functions for the A1 surface electronic state at $\epsilon_{F}$ in the $\overline{\mathrm{A} \Gamma}$ (a) and $\overline{\mathrm{AL}}$ (b) symmetry directions. The calculated spectra are quite different, demonstrating that peak positions in the calculated $\alpha^{2} F(\omega)$ depend on the momentum position of electronic state at the Fermi energy line. In the case of the $\overline{\mathrm{AL}}$ direction, there are peaks at 60 and above $75 \mathrm{meV}$ that are determined for the most part by surface modes around $\overline{\mathrm{M}}$ and contribute strongly to intraband transitions. For electronic states in the $\overline{\mathrm{A} \Gamma}$ direction, the peaks at $60 \mathrm{meV}$ are substantially suppressed for lack of final states; however, high-energy modes still participate in the $e$-ph coupling. With respect to the low-frequency part of the calculated $\alpha^{2} F(\omega)$, it is far from Debye-like and is essentially zero in both cases. The exception is a sharp peak at $\approx 20 \mathrm{meV}$ in the upper panel (the state in the $\overline{\mathrm{A} \Gamma}$ direction), which is related to intraband coupling via surface vibrations from the $\overline{\Gamma A}$ direction. The wave vectors of these modes are fully determined by the momentum position of the electronic state, which results in a rather narrow peak. Because of the $1 / \omega$ weighting in the definition of $\lambda$, the assumption of a Debye-like low-frequency part can easily lead to a significant overestimate of $\lambda$.

\section{SUMMARY}

We have presented an analysis of the $e$-ph interaction in surface electronic states on $\mathrm{Be}(10 \overline{1} 0)$, using $a b$ initio calculations. It is found that the strength of the coupling usually varies between 0.30 and 0.55 with the momentum and energy position of an electronic state. The exceptions are the upper surface electronic state at $\overline{\mathrm{A}}$ at $\epsilon \leqslant \epsilon_{F}$ and the surface electronic states with a weak localization at the surface, M2 and $\Gamma$, for which $\lambda(\epsilon, \mathbf{k})$ is quite small. The $e$-ph interaction in the lower surface state at $\overline{\mathrm{A}}, \mathrm{A} 2$, is largely determined by the intraband scattering and the coupling to other surface electronic states. The intraband scattering is also responsible for the enhancement of the $e$-ph coupling in the upper surface state at $\overline{\mathrm{A}}, \mathrm{A} 1$, with decreasing binding energy and at $\epsilon \geqslant \epsilon_{F}$.

Our work also shows that the difference in the strength of the $e$-ph coupling in the upper and lower surface states at $\overline{\mathrm{A}}$ is directly related to their different energy positions in the projected band gap. Because A1 lies in the middle of the wide band gap, only short-wavelength lattice vibrations contribute substantially to the scattering of electrons. The probability of such transitions mediated by large wave-vector phonons is rather low. The participation of the low-energy phonon modes is negligible up to $\sim 30 \mathrm{meV}$ due to a lack of final electronic states. As a result, the Debye model fails to describe the lowenergy part of the spectral function for A1, which is very important in the definition of $\lambda$.

The calculated $e$-ph coupling strength for the lower surface state at $\overline{\mathrm{A}}$ agrees well with experimental findings. However, there is a severe discrepancy for the upper surface state between calculated and experimentally derived $\lambda(\epsilon, \mathbf{k})$. Part of the discrepancy could be due to the use of inappropriate models in the analysis of the experimental data. In view of the unusual shape of $\alpha^{2} F(\omega)$ for the A1 state, a reexamination of these experiments taking this specific feature of the spectral function into account would be desirable.

\section{ACKNOWLEDGMENTS}

We acknowledge financial support of the University of the Basque Country UPV/EHU (Grant No. GIC071T36607), the Departamento de Educación del Gobierno Vasco, and the Spanish Ministerio de Ciencia y Tecnología (MCyT) (Grant No. FIS 2007-66711-C01-01).
${ }^{1}$ G. Grimvall, The Electron-Phonon Interaction in Metals (NorthHolland, New York, 1981).

${ }^{2}$ J. Kröger, Rep. Prog. Phys. 69, 899 (2006).
${ }^{3}$ R. Haight, Surf. Sci. Rep. 21, 275 (1995).

${ }^{4}$ B. Hellsing, A. Eiguren, and E. V. Chulkov, J. Phys. Condens. Matter 14, 5959 (2002). 
${ }^{5}$ E. W. Plummer, J. Shi, S. -J. Tang, E. Rotenberg, and S. D. Kevan, Prog. Surf. Sci. 74, 251 (2003).

${ }^{6} \mathrm{P}$. M. Echenique, R. Berndt, E. V. Chulkov, Th. Fauster, A. Goldmann, and U. Höfer, Surf. Sci. Rep. 52, 219 (2004).

${ }^{7}$ E. V. Chulkov, A. G. Borisov, J. P. Gauyacq, D. Sánchez-Portal, V. M. Silkin, V. P. Zhukov, and P. M. Echenique, Chem. Rev. 106, 4160 (2006).

${ }^{8}$ P. Saalfrank, Chem. Rev. 106, 4116 (2006).

${ }^{9}$ J. Kröger, Appl. Phys. A 87, 345 (2007).

${ }^{10} \mathrm{Ph}$. Hofmann, I. Yu. Sklyadneva, E. D. L. Rienks, and E. V. Chulkov, New J. Phys. 11, 125005 (2009).

${ }^{11}$ B. A. McDougall, T. Balasubramanian, and E. Jensen, Phys. Rev. B 51, 13891 (1995).

${ }^{12}$ F. Theilmann, R. Matzdorf, G. Meister, and A. Goldmann, Phys. Rev. B 56, 3632 (1997).

${ }^{13}$ A. Eiguren, B. Hellsing, F. Reinert, G. Nicolay, E. V. Chulkov, V. M. Silkin, S. Hüfner, and P. M. Echenique, Phys. Rev. Lett. 88, 066805 (2002).

${ }^{14}$ C. Kirkegaard, T. K. Kim, and Ph. Hofmann, New J. Phys. 7, 99 (2005).

${ }^{15}$ T. Valla, A. V. Fedorov, P. D. Johnson, and S. L. Hulbert, Phys. Rev. Lett. 83, 2085 (1999).

${ }^{16}$ R. Matzdorf, G. Meister, and A. Goldmann, Phys. Rev. B 54, 14807 (1996).

${ }^{17}$ J. E. Gayone, S. V. Hoffmann, Z. Li, and Ph. Hofmann, Phys. Rev. Lett. 91, 127601 (2003).

${ }^{18}$ T. K. Kim, T. S. Sørensen, E. Wolfring, H. Li, E. V. Chulkov, and Ph. Hofmann, Phys. Rev. B 72, 075422 (2005).

${ }^{19}$ T. Balasubramanian, P. -A. Glans, and L. I. Johansson, Phys. Rev. B 61, 12709 (2000).

${ }^{20}$ T. Balasubramanian, L. I. Johansson, P. -A. Glans, C. Virojanadara, V. M. Silkin, E. V. Chulkov, and P. M. Echenique, Phys. Rev. B 64, 205401 (2001).

${ }^{21}$ S. J. Tang, Ismail, P. T. Sprunger, and E. W. Plummer, Phys. Rev. B 65, 235428 (2002).

${ }^{22}$ M. Hengsberger, R. Frésard, D. Purdie, P. Segovia, and Y. Baer, Phys. Rev. B 60, 10796 (1999); M. Hengsberger, D. Purdie, P. Segovia, M. Garnier, and Y. Baer, Phys. Rev. Lett. 83, 592 (1999).
${ }^{23}$ S. LaShell, E. Jensen, and T. Balasubramanian, Phys. Rev. B 61, 2371 (2000).

${ }^{24}$ J. Shi, S. -J. Tang, B. Wu, P. T. Sprunger, W. L. Yang, V. Brouet, X. J. Zhou, Z. Hussain, Z. -X. Shen, Z. Zhang, and E. W. Plummer, Phys. Rev. Lett. 92, 186401 (2004).

${ }^{25}$ R. A. Bartynski, E. Jensen, T. Gustafsson, and E. W. Plummer, Phys. Rev. B 32, 1921 (1985).

${ }^{26}$ E. V. Chulkov, V. M. Silkin, and E. N. Shirykalov, Surf. Sci. 188, 287 (1987).

${ }^{27}$ A. Eiguren, S. de Gironcoli, E. V. Chulkov, P. M. Echenique, and E. Tosatti, Phys. Rev. Lett. 91, 166803 (2003).

${ }^{28}$ A. Leonardo, I. Yu. Sklyadneva, P. M. Echenique, and E. V. Chulkov, Surf. Sci. 600, 3715 (2006).

${ }^{29} \mathrm{Ph}$. Hofmann, R. Stumpf, V. M. Silkin, E. V. Chulkov, and E. W. Plummer, Surf. Sci. 355, L278 (1996).

${ }^{30} \mathrm{Ph}$. Hofmann and E. W. Plummer, Surf. Sci. 377-379, 330 (1997).

${ }^{31}$ I. Yu. Sklyadneva, E. V. Chulkov, W. -D. Schöne, V. M. Silkin, R. Keyling, and P. M. Echenique, Phys. Rev. B 71, 174302 (2005).

${ }^{32}$ W. Kohn and L. J. Sham, Phys. Rev. 140, A1133 (1965).

${ }^{33}$ L. Hedin and B. I. Lundqwist, J. Phys. C 4, 20604 (1971).

${ }^{34}$ R. Heid and K. -P. Bohnen, Phys. Rev. B 60, R3709 (1999).

${ }^{35}$ D. Vanderbilt, Phys. Rev. B 32, 8412 (1985).

${ }^{36}$ S. G. Louie, S. Froyen, and M. L. Cohen, Phys. Rev. B 26, 1738 (1982).

${ }^{37}$ K. J. H. Mackay and N. A. Hill, J. Nucl. Mater. 8, 263 (1963).

${ }^{38}$ G. M. Eliashberg, Sov. Phys. JETP 16, 780 (1962) [Zh. Eksp. Teor. Fiz. 43, 1005 (1962)].

${ }^{39}$ P. B. Allen and M. L. Cohen, Phys. Rev. 187, 525 (1969).

${ }^{40} \mathrm{Ph}$. Hofmann, K. Pohl, R. Stumpf, and E. W. Plummer, Phys. Rev. B 53, 13715 (1996).

${ }^{41}$ M. Lazzeri and S. de Gorincoli, Surf. Sci. 454-456, 442 (2000).

${ }^{42}$ S. J. Tang, H. -T. Jeng, C. -S. Hsue, Ismail, P. T. Sprunger, and E. W. Plummer, Phys. Rev. B 77, 045405 (2008).

${ }^{43}$ V. M. Silkin and E. V. Chulkov, Sov. Phys. Solid State 37, 1540 (1995) [Fiz. Tverd. Tela (S.-Petersburg) 37, 2795 (1995)].

${ }^{44}$ R. Heid and K. -P. Bohnen, Phys. Rep. 387, 151 (2003). 